\title{
Impact of Temporary Portocaval Shunting and Initial Arterial Reperfusion in Orthotopic Liver Transplantation
}

\author{
Lars Cornelis Pietersen, ${ }^{1}$ Elise Sarton, ${ }^{2}$ Ian Alwayn, ${ }^{1}$ Hwai-Ding Lam, ${ }^{1}$ Hein Putter, ${ }^{3}$ \\ Bart van Hoek, ${ }^{4 *}$ and Andries Erik Braat ${ }^{1 *}$ \\ ${ }^{1}$ Division of Transplantation, Departments of Surgery, ${ }^{2}$ Anesthesiology, ${ }^{3}$ Medical Statistics, and ${ }^{4}$ Gastroenterology and \\ Hepatology, Leiden University Medical Center, Leiden, the Netherlands
}

\begin{abstract}
The use of a temporary portocaval shunt (TPCS) as well as the order of reperfusion (initial arterial reperfusion [IAR] versus initial portal reperfusion) in orthotopic liver transplantation (OLT) is controversial and, therefore, still under debate. The aim of this study was to evaluate outcome for the 4 possible combinations (temporary portocaval shunt with initial arterial reperfusion $\left[\mathrm{A}+\mathrm{S}_{+}\right]$, temporary portocaval shunt with initial portal reperfusion, no temporary portocaval shunt with initial arterial reperfusion, and no temporary portocaval shunt with initial portal reperfusion) in a center-based cohort study, including liver transplantations (LTs) from both donation after brain death and donation after circulatory death (DCD) donors. The primary outcome was the perioperative transfusion of red blood cells (RBCs), and the secondary outcomes were operative time and patient and graft survival. Between January 2005 and May 2017, all first OLTs performed in our institution were included in the 4 groups mentioned. With IAR and TPCS, a significantly lower perioperative transfusion of RBCs was seen $(P<0.001)$ as well as a higher number of recipients without any transfusion of RBCs $(P<0.001)$. A multivariate analysis showed laboratory Model for End-Stage Liver Disease (MELD) score $(P<0.001)$ and IAR $(P=0.01)$ to be independent determinants of the transfusion of RBCs. When comparing all groups, no statistical difference was seen in operative time or in 1-year patient and graft survival rates despite more LTs with a liver from a DCD donor in the $\mathrm{A}+\mathrm{S}+$ group $(P=0.005)$. In conclusion, next to a lower laboratory MELD score, the use of IAR leads to a significantly lower need for perioperative blood transfusion. There was no significant interaction between IAR and TPCS. Furthermore, the use of a TPCS and/or IAR does not lead to increased operative time and is therefore a reasonable alternative surgical strategy.
\end{abstract}

Liver Transplantation 25 1690-1699 2019 AASLD.

Received December 28, 2018; accepted June 6, 2019.

During orthotopic liver transplantation (OLT), clamping and sectioning of the portal vein (PV) from the native liver induces splanchnic venous congestion, intestinal edema, bacterial translocation, and

Abbreviations: $A+S+$, temporary portocaval shunt with initial arterial reperfusion; $A+S-$, no temporary portocaval shunt with initial arterial reperfusion; $A-S+$, temporary portocaval shunt with initial portal reperfusion; $A-S-$, no temporary portocaval shunt with initial portal reperfusion; ALT, alanine aminotransferase; AST, aspartate aminotransferase; BAR, balance of risk; BMI, body mass index; CIT, cold ischemia time; $D B D$, donation after brain death; $D C D$, donation after circulatory death; DRM, donor-recipient model; ET-DRI, Eurotransplant donor risk index; FFP, fresh frozen plasma; $G G T$, gamma-glutamyltransferase; IAR, initial arterial reperfusion; INR, international normalized ratio; LT, liver transplantation; MELD, Model for End-Stage Liver Disease; NR, not reported; OLT, orthotopic liver transplantation; PTT, partial thromboplastin time; $P V$, portal accumulation of noxious elements. ${ }^{(1,2)}$ The use of a temporary portocaval shunt (TPCS) may prevent these complications by alleviating gut edema, reducing bleeding with reduction of portal venous pressure, and improving hemodynamic stability. ${ }^{(3)}$

After the introduction of TPCSs in 1993 by Tzakis et al., ${ }^{(4)}$ the evidence of its benefit has been controversial. Several retrospective studies have shown better intraoperative hemodynamic parameters, a decrease in the incidence of reperfusion syndrome, ${ }^{(5,6)}$ and better graft survival ${ }^{(7)}$ by using a TPCS. However, other studies $^{(3,8)}$ showed no effect of a TPCS on intraoperative transfusion of red blood cells (RBCs). Therefore, potential advantages of the TPCS remain questionable, and its use is still debated. ${ }^{(9-11)}$

Several older, small, prospective studies that were nonrandomized, randomized, and retrospective 
investigated initial arterial reperfusion (IAR) $)^{(12-17)}$ and showed controversial results on outcomes after liver transplantation (LT). Thus, the ideal sequence of reperfusion is an issue that is still debated. The increased demand for LT has led to the increased use of extended criteria donor livers, specifically from donation after circulatory death (DCD) donors. ${ }^{(18-20)}$ In contrast to LT with donation after brain death (DBD) organs, DCD LT is known to have an increased risk for posttransplantation complications, especially early allograft dysfunction, acute kidney injury, and nonanastomotic biliary strictures. ${ }^{(21)}$ Little is known about the effect of a TPCS or IAR in DCD LT.

The aim of this study was to evaluate perioperative blood loss, hepatic injury, operative time, and outcomes for LT with or without TPCS and/or IAR in a retrospective center-based cohort study including both DBD and DCD LTs. This study has received approval by the institutional review committee.

\section{Patients and Methods}

\section{PATIENTS}

Between January 2005 and May 2017, all LTs at the Leiden University Medical Center, Leiden, the Netherlands, were included in this study. Recipients who received a domino, split, or auxiliary LT or a retransplantation were excluded. Clinical information was obtained from a prospectively collected database.

vein; $R B C$, red blood cell; $S D$, standard deviation; SE, standard error; sRRI, simplified recipient risk index; TPCS, temporary portocaval shunt; WIT, warm ischemia time.

Address reprint requests to Lars Cornelis Pietersen, M.D., Division of Transplantation, Department of Surgery, Leiden University Medical Center, Albinusdreef 2, 2333 ZA Leiden, the Netherlands. Telephone: +3171-5266188; FAX: +31-71-5266952; E-mail: l.c.pietersen@lumc.nl

*These authors contributed equally to this work.

Copyright (C) 2019 by the American Association for the Study of Liver Diseases.

View this article online at wileyonlinelibrary.com.

DOI 10.1002/lt.25592

Potential conflict of interest: Nothing to report.

Correction Statement: Correction added on October 11, 2019 after first online publication: In Fig. 1 , the $x$-axis was set incorrectly as $A-S+$, $A+S-, A-S+, A+S+$. It has been updated to read: $A-S-, A+S-, A-S+$, $A+S+$. We apologize to the author and to our readers for this error.
Covariates included donor demographics, recipient demographics, pretransplant information, intraoperative data, and postoperative outcomes.

Laboratory Model for End-Stage Liver Disease (MELD) scores were included in the recipient analysis. If necessary, the original patient notes were reviewed for missing information. The Eurotransplant donor risk index (ET-DRI), simplified recipient risk index (sRRI), combined donor-recipient model (DRM), and balance of risk (BAR) scores were calculated as described previously. ${ }^{(22-24)}$ The peak value of aspartate aminotransferase (AST) and alanine aminotransferase (ALT) during the first 7 days after transplantation was used as a marker of ischemia/reperfusion injury. ${ }^{(25)}$

\section{DEFINITION OF PERIOPERATIVE BLOOD LOSS}

Mild blood loss requiring transfusion may often be ongoing after surgery. Therefore, perioperative blood loss was defined as the need for transfusion of RBCs during the first 24 hours after the start of surgery. Moreover, Cell Saver (LivaNova, London, UK) volume and fresh frozen plasma (FFP) transfusion were also noted.

\section{OPERATIVE TECHNIQUES OF RECIPIENT SURGERY}

Briefly, the standard incision and exposure was followed by the dissection of the hepatoduodenal ligament and by liver mobilization. Since May 2010, a change in center protocol was incorporated, which consisted of the use of a TPCS prior to mobilization and removal of the native liver. The TPCS consisted of an end-to-side anastomosis of the PV to the inferior vena cava at the level of the renal veins. After insertion of the liver graft, a side-to-side caval anastomosis was performed. Shortly after the introduction of a TPCS, IAR was introduced. In the historic control group, the portal anastomosis was directly followed by portal reperfusion of the liver. Thereafter, arterial anastomosis and reperfusion followed. Since the change in protocol, the arterial anastomosis has been directly followed by arterial reperfusion of the liver. Hereafter, portal anastomosis and reperfusion followed. Just before portal reconstruction, the TPCS was divided using a vascular endo GIA stapling device (Medtronic, Minneapolis, $\mathrm{MN}$ ), after which a standard end-to-end portal anastomosis was performed. Finally, biliary reconstruction was performed, preferably with a duct-to-duct anastomosis. 


\section{STATISTICAL ANALYSIS}

Continuous variables were presented as mean and standard deviation (SD), or median (range) and SD, whereas categorical variables were presented as $\mathrm{n}(\%)$. Categorical variables were compared with the Pearson's chi-square test. Characteristics of the donor, transplantation, and recipient were analyzed using 1-way analysis of variance. Perioperative blood loss and peak value of AST and ALT were analyzed by using the Kruskal-Wallis test. Patient and graft survival rates, noncensored for death, were analyzed by KaplanMeier estimation with a log-rank test for differences.

\section{MULTIVARIATE ANALYSIS}

To analyze the influence of covariates on blood loss, a multivariate logistic regression was performed using donor, transplantation, and recipient covariates that were most likely to influence blood loss. A possible interaction between TPCS and IAR was also examined in a secondary model. The level of significance was set at 0.05. Statistical analyses were performed using SPSS, version 25.0 for Windows (SPSS Inc., Chicago, IL).

\section{Results}

In total, 365 patients received a LT between January 2005 and May 2017. There were 60 recipients who were excluded due to receiving either a domino LT $(\mathrm{n}=3)$, split-liver transplantation $(\mathrm{n}=2)$, auxiliary LT $(n=11)$, or retransplantation $(n=44)$. The use of a TPCS and the order of reperfusion could not be traced in 2 patients, who were therefore excluded from further analysis. Of the 303 recipients included in the study, 156 (51\%) received no temporary portocaval shunt with initial portal reperfusion (A-S-); $15(5 \%)$ received no temporary portocaval shunt with initial arterial reperfusion $(\mathrm{A}+\mathrm{S}-) ; 41$ (14\%) received temporary portocaval shunt with initial portal reperfusion $(\mathrm{A}-\mathrm{S}+)$; and $91(30 \%)$ received temporary portocaval shunt with initial arterial reperfusion $(\mathrm{A}+\mathrm{S}+)$.

TABLE 1. Donor, Transplantation, and Recipient Characteristics

\begin{tabular}{|c|c|c|c|c|c|}
\hline & $A-S-(n=156)$ & $A+S-(n=15)$ & $A-S+(n=41)$ & $A+S+(n=91)$ & $P$ Value \\
\hline Donor age, years & $48 \pm 15$ & $46 \pm 20$ & $46 \pm 15$ & $45 \pm 16$ & 0.56 \\
\hline Recipient age, years & $54 \pm 10$ & $48 \pm 15$ & $52 \pm 14$ & $55 \pm 10$ & 0.10 \\
\hline Recipient BMI, $\mathrm{kg} / \mathrm{m}^{2}$ & $27 \pm 5$ & $26 \pm 4$ & $25 \pm 4$ & $26 \pm 4$ & 0.36 \\
\hline Medical history, \% & & & & & 0.16 \\
\hline Metabolic disease & 4 & 0 & 7 & 4 & \\
\hline Acute liver disease & 5 & 0 & 10 & 9 & \\
\hline Cholestatic liver disease & 11 & 40 & 17 & 13 & \\
\hline Alcoholic liver disease & 19 & 13 & 5 & 18 & \\
\hline Malignancy & 32 & 20 & 46 & 36 & \\
\hline Hepatitis B & 2 & 0 & 2 & 2 & \\
\hline Hepatitis C & 4 & 0 & 0 & 4 & \\
\hline Other cirrhosis & 15 & 7 & 7 & 9 & \\
\hline Other/unknown & 8 & 20 & 6 & 6 & \\
\hline DCD liver grafts, \% & 33 & 27 & 32 & 54 & 0.005 \\
\hline Portal hypertension, \% & 25 & 43 & 44 & 35 & 0.10 \\
\hline ET-DRI & $1.81 \pm 0.3$ & $1.73 \pm 0.4$ & $1.70 \pm 0.3$ & $1.83 \pm 0.3$ & 0.18 \\
\hline sRRI & $1.96 \pm 0.6$ & $1.80 \pm 0.4$ & $1.94 \pm 0.7$ & $2.08 \pm 0.7$ & 0.26 \\
\hline DRM & $1.40 \pm 0.1$ & $1.35 \pm 0.1$ & $1.38 \pm 0.1$ & $1.42 \pm 0.1$ & 0.11 \\
\hline BAR score & $5.983 \pm 4.2$ & $5.60 \pm 4.1$ & $7.02 \pm 5.3$ & $6.38 \pm 4.7$ & 0.42 \\
\hline Laboratory MELD & $15 \pm 8$ & $15 \pm 9$ & $16 \pm 11$ & $16 \pm 9$ & 0.75 \\
\hline CIT, minutes & $566 \pm 124$ & $552 \pm 168$ & $522 \pm 108$ & $540 \pm 127$ & 0.18 \\
\hline WIT, minutes & $34 \pm 8$ & $42 \pm 11$ & $38 \pm 14$ & $39 \pm 12$ & $<0.001$ \\
\hline Operative time, minutes & $338 \pm 82$ & $355 \pm 99$ & $358 \pm 93$ & $322 \pm 90$ & 0.12 \\
\hline
\end{tabular}

NOTE: Data are presented as mean \pm SD. Bolded values are significant. 


\section{DONOR, TRANSPLANT, AND RECIPIENT CHARACTERISTICS}

Tables 1-4 show the basic donor, transplantation, and recipient characteristics of all groups. The $\mathrm{A}+\mathrm{S}+$ group consisted of a significantly higher percentage of DCD LTs compared with the other groups (33\% in the A-Sgroup versus $27 \%$ in the $\mathrm{A}+\mathrm{S}-$ group versus $32 \%$ in the $\mathrm{A}-\mathrm{S}+$ group versus $54 \%$ in the $\mathrm{A}+\mathrm{S}+$ group; $P=0.005$ ). The warm ischemia time was significantly shorter in the A-S- group compared with the other groups

TABLE 2. Post Hoc Analysis for WIT and DCD LTs

\begin{tabular}{lcc} 
& \multicolumn{2}{c}{$P$ Value } \\
\cline { 2 - 3 } Operation Technique & WIT & DCD \\
\hline A-S- & & \\
A+S- & $\mathbf{0 . 0 0 5}$ & 0.78 \\
A-S+ & $\mathbf{0 . 0 2}$ & 0.17 \\
A+S+ & $<\mathbf{0 . 0 0 1}$ & $\mathbf{0 . 0 0 2}$ \\
A+S- & & \\
A-S- & $\mathbf{0 . 0 0 5}$ & 0.78 \\
A-S+ & 0.24 & 0.79 \\
A+S+ & 0.31 & $\mathbf{0 . 0 0 9}$ \\
A-S+ & & \\
A-S- & $\mathbf{0 . 0 2}$ & 0.17 \\
A+S- & 0.24 & 0.79 \\
A+S+ & 0.71 & $\mathbf{0 . 0 3}$ \\
A+S+ & & \\
A-S- & $<0.001$ & $\mathbf{0 . 0 0 2}$ \\
A+S- & 0.31 & $\mathbf{0 . 0 0 9}$ \\
A-S+ & 0.71 & $\mathbf{0 . 0 3}$
\end{tabular}

NOTE: Bolded values are significant.
$(34 \pm 8$ minutes in the $\mathrm{A}-\mathrm{S}-$ group versus $42 \pm 11$ minutes in the $\mathrm{A}+\mathrm{S}-$ group versus $38 \pm 14$ minutes in the $\mathrm{A}-\mathrm{S}+$ group versus $39 \pm 12$ minutes in the $\mathrm{A}+\mathrm{S}+$ group; $P<0.001$ ). Other donor, transplantation, and recipient characteristics did not significantly differ among the groups. Also, the mean operative time did not significantly differ among the groups $(P=0.12)$.

\section{BLOOD LOSS}

Tables 3 and 4 show the hematological and coagulation parameters of all groups preoperatively and the number of packed RBCs, FFP, and Cell Saver fluid (mL) transfused during the first 24 hours from incision. The partial thromboplastin time (PTT) in the A-S- group was significantly shorter compared with the other groups $(P=0.04)$. Other preoperative hematological and coagulation parameters did not differ between both groups. The median number of packed RBCs transfused in the A-S- group was $6 \pm 7$ units (range, $0-33$ units) versus $2 \pm 6$ units (range, $0-19$ units) in the $\mathrm{A}+\mathrm{S}$ - group, $5 \pm 6$ units (range, $0-30$ units) in the $\mathrm{A}-\mathrm{S}+$ group, and $2 \pm 5$ units (range, $0-23$ units) in the $\mathrm{A}+\mathrm{S}+$ group $(P<0.001$; Fig. 1). When analyzing outcomes based on TPCS status alone, the use of a TPCS showed a significantly lower median number of transfused RBCs compared with the group who did not receive a TPCS (3 versus 6 units; $P<0.001)$.

Of the recipients in the $\mathrm{A}+\mathrm{S}+$ group, $31 \%$ did not receive any $\mathrm{RBC}$ s perioperatively, versus $28 \%$ in the $\mathrm{A}-\mathrm{S}+$ group, $27 \%$ in the $\mathrm{A}+\mathrm{S}-$ group, and $8 \%$ in the A-S- group $(P<0.001)$. The mean amount of FFP transfused as well as the mean volume of Cell Saver transfused did not differ among the groups.

TABLE 3. Hematological, Coagulation, and Transfusion Parameters Before, During, and After Surgery

\begin{tabular}{|c|c|c|c|c|c|}
\hline & A-S- $(n=156)$ & $A+S-(n=15)$ & $A-S+(n=41)$ & $A+S+(n=91)$ & $P$ Value \\
\hline INR before surgery & $1.35 \pm 0.41$ & $1.39 \pm 0.48$ & $1.51 \pm 0.60$ & $1.40 \pm 0.44$ & 0.25 \\
\hline $\begin{array}{l}\text { Platelet count before } \\
\text { surgery, } 10^{9} / \mathrm{L}\end{array}$ & $110 \pm 80$ & $164 \pm 118$ & $123 \pm 98$ & $119 \pm 82$ & 0.12 \\
\hline PTT before surgery, seconds & $18 \pm 7$ & $20 \pm 7$ & $21 \pm 9$ & $20 \pm 7$ & 0.04 \\
\hline Fibrinogen before surgery, g/L & $2.9 \pm 1.4$ & $3.5 \pm 1.5$ & $3.7 \pm 5.6$ & $2.8 \pm 1.4$ & 0.15 \\
\hline FFP, units & $9 \pm 9$ & $7 \pm 9$ & $10 \pm 9$ & $8 \pm 9$ & 0.45 \\
\hline Packed RBCs, units & $6 \pm 7$ & $2 \pm 6$ & $5 \pm 6$ & $2 \pm 5$ & $<0.001$ \\
\hline Cell Saver, mL* & $1072 \pm 1158$ & $918 \pm 1006$ & $887 \pm 949$ & $1418 \pm 1231$ & 0.11 \\
\hline No packed RBCs used, \% & 8 & 27 & 28 & 31 & $<0.001$ \\
\hline
\end{tabular}

NOTE: Data are presented as mean or median $\pm \mathrm{SD}$. Bolded values are significant.

*Cell Saver values were missing for 99 recipients. 
TABLE 4. Post Hoc Analysis for Packed Cells Transfused

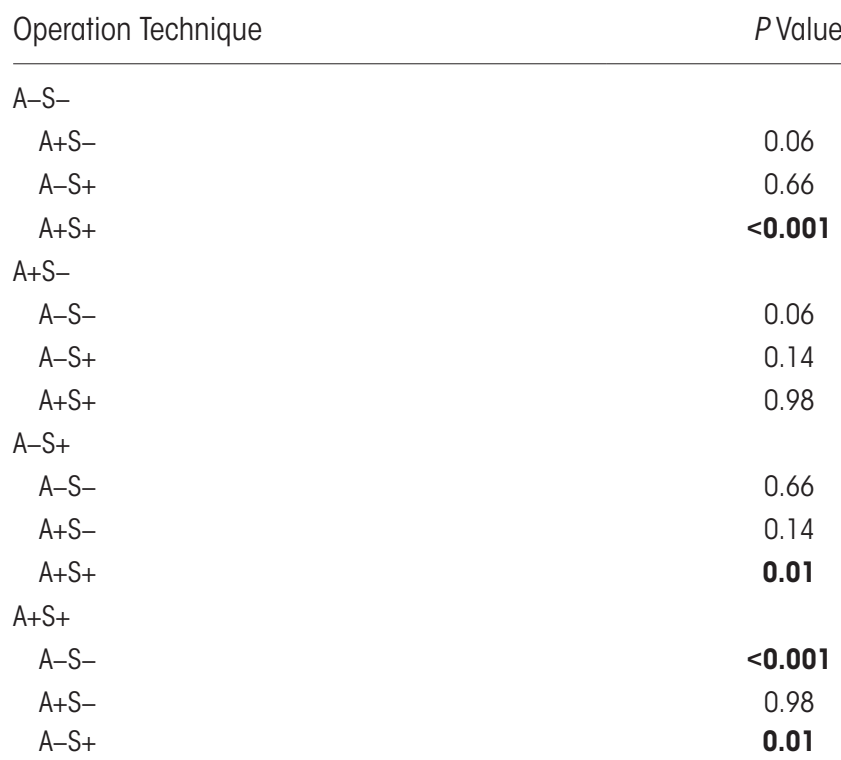

NOTE: Bolded values are significant.

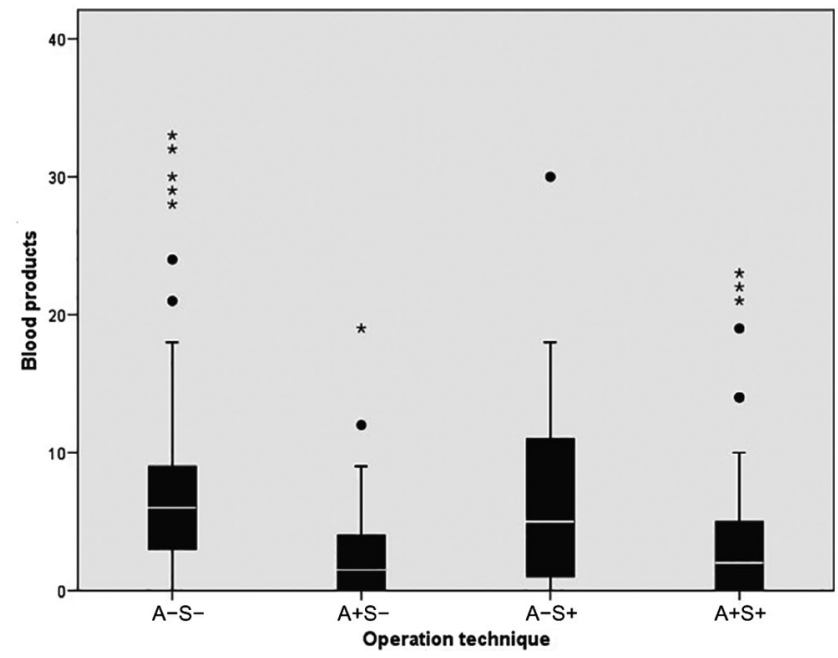

FIG. 1. Boxplot showing the distribution of RBCs transfused perioperatively among the 4 groups. ${ }^{\bullet}=$ outliers, ${ }^{*}=$ extreme outliers.

\section{POSTTRANSPLANTATION PEAK OF AST AND ALT}

Table 5 shows the median peak of AST and ALT during the first 7 days after transplantation. The median peak of AST and ALT for all groups did not significantly differ (AST, $P=0.89$; ALT, $P=0.92$ ).

When analyzing only DCD LT, the median peak of AST and ALT also did not significantly differ for all groups (AST, $P=0.13$; ALT, $P=0.31$ ). Also, when performing a Kaplan-Meier analysis with log-rank testing on biliary complications, no significant difference was seen among all groups $(P=0.57)$ even though the $\mathrm{A}+\mathrm{S}+$ group consisted of significantly more $\mathrm{DCD}$ LTs $(P=0.005)$.

\section{POSTOPERATIVE COMPLICATIONS}

When comparing postoperative complications, no statistical difference was seen in Clavien-Dindo complications that were of a grade $\geq 3(P=0.85$; Table 6$)$.

\section{PATIENT AND GRAFT SURVIVAL}

Figure 2 shows the 1-year patient survival. No significant difference was found when comparing all of the groups. Figure 3 shows the 1-year graft survival noncensored for death. No significant difference was found in 1-year patient survival or in 1-year graft survival noncensored for death.

\section{MULTIVARIATE ANALYSIS}

When performing multivariate logistic regression of all covariates included, the laboratory MELD score $(P<0.001)$ and IAR $(P=0.01)$ were identified as individual determinants for increased transfusion of RBCs. Interestingly, TPCS did not show a significant difference $(P=0.78)$. Furthermore, IAR and TPCS did not show a significant statistical interaction $(P=0.54$; Table 7$)$. All other potential determinants did not show a significant difference.

\section{Discussion}

This cohort study demonstrates that LT with IAR and TPCS was associated with less perioperative transfusion of RBCs. Furthermore, multivariate analysis showed that laboratory MELD and IAR were individual determinants on the number of RBCs transfused.

To our knowledge, this study is the first to use multivariate analysis to determine individual determinants of perioperative transfusion of RBCs in LT. When performing univariate analysis, a significant beneficial effect of IAR and TPCS on transfusion of RBCs during LT was shown $(P<0.001)$. However, multivariate analysis showed only laboratory MELD and IAR to be individual determinants of perioperative 
TABLE 5. Peak of AST and ALT During the First 7 Days After Transplantation for the Whole Population as Well as for DCD LT Separately

\begin{tabular}{|c|c|c|c|c|c|}
\hline & $A-S-(n=156)$ & $A+S-(n=15)$ & $A-S+(n=41)$ & $A+S+(n=91)$ & $P$ Value \\
\hline AST, U/L & $1218 \pm 2832$ & $1332 \pm 1556$ & $1096 \pm 2974$ & $1126 \pm 2573$ & 0.89 \\
\hline ALT, U/L & $775 \pm 1863$ & $742 \pm 1285$ & $688 \pm 1804$ & $777 \pm 1713$ & 0.92 \\
\hline \multicolumn{6}{|l|}{ DCD LT } \\
\hline AST, U/L & $2199 \pm 4017$ & $3833 \pm 1900$ & $1161 \pm 4663$ & $1309 \pm 2893$ & 0.13 \\
\hline ALT, U/L & $1525 \pm 2326$ & $2011 \pm 2000$ & $947 \pm 2877$ & $925 \pm 1814$ & 0.31 \\
\hline
\end{tabular}

NOTE: Data are given as median $\pm \mathrm{SD}$.

TABLE 6. Clavien-Dindo Complications Grade $\geq 3$

\begin{tabular}{|c|c|c|c|c|c|}
\hline & $A-S-(n=156)$ & $A+S-(n=15)$ & $A-S+(n=41)$ & $A+S+(n=91)$ & $P$ Value \\
\hline \multicolumn{6}{|c|}{$\begin{array}{l}\text { Clavien-Dindo } \\
\text { classification grade }\end{array}$} \\
\hline $3 a$ & $18(12)$ & $2(13)$ & $4(10)$ & $10(11)$ & \\
\hline $3 b$ & 27 (17) & $3(20)$ & $5(12)$ & $18(20)$ & \\
\hline $4 a$ & $8(5)$ & $0(0)$ & $4(10)$ & $8(9)$ & \\
\hline $4 b$ & $7(4)$ & $0(0)$ & $1(2)$ & $1(1)$ & \\
\hline 5 & $5(3)$ & $1(7)$ & $1(2)$ & $3(3)$ & \\
\hline Total & $65(42)$ & $6(40)$ & $15(37)$ & $40(44)$ & 0.85 \\
\hline
\end{tabular}

NOTE: Data are given as $\mathrm{n}(\%)$.

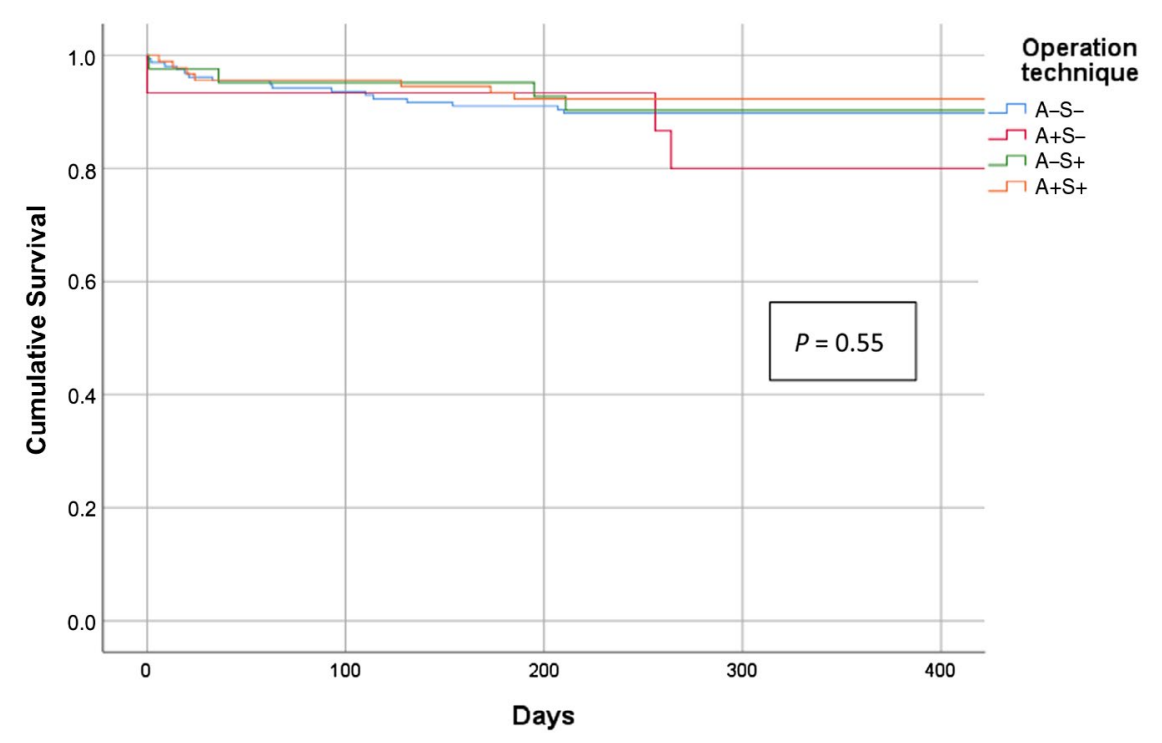

FIG. 2. The 1-year patient survival curve.

transfusion of RBCs, whereas the interaction between IAR and TPCS was low $(P=0.54)$. The beneficial effect of a TPCS, by using univariate analysis, has been described before (Table 8). ${ }^{(7,26-28)}$ These studies do not describe the order of reperfusion used during transplantation, although we assume that portal reperfusion was first. A meta-analysis performed by Pratschke et al. showed a significant beneficial effect of a TPCS on operative blood loss, but the $\mathrm{I}^{2}$ values indicated substantial heterogeneity among the studies $(P<0.05)$, which could bring a potential bias to the results. ${ }^{(29)}$ 


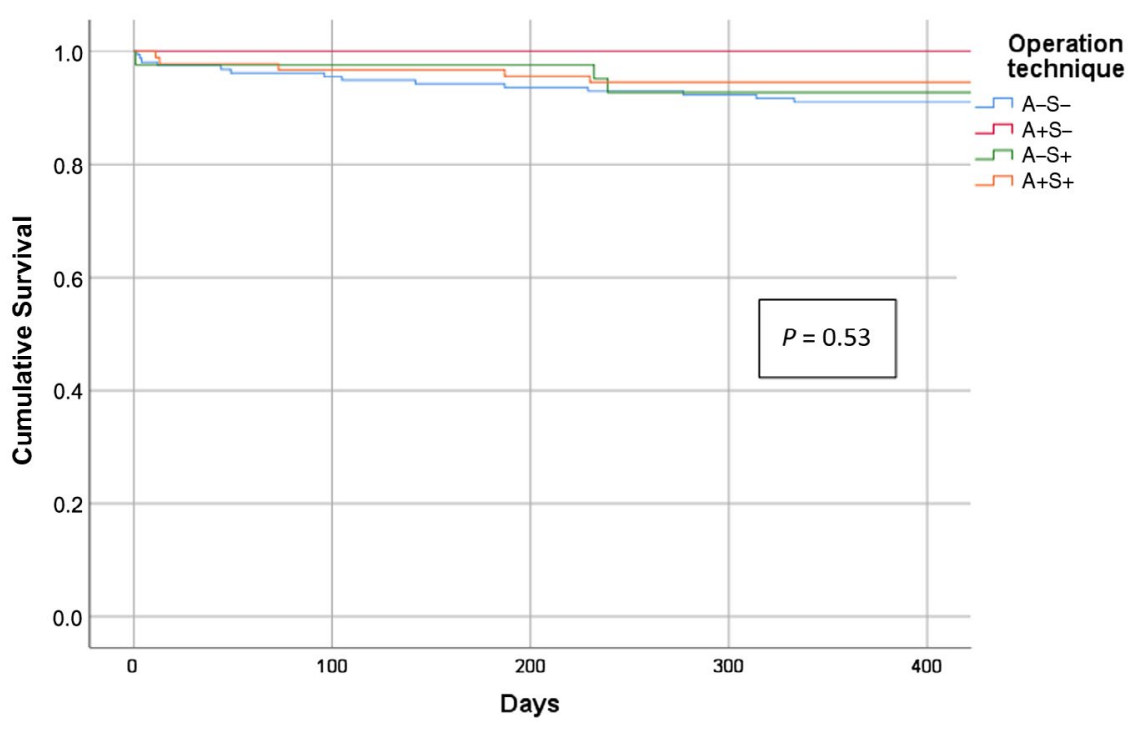

FIG. 3. The 1-year graft survival curve noncensored for death.

TABLE 7. Multivariate Analysis on Perioperative Transfusion of RBCs

\begin{tabular}{lccc} 
& $B$ & SE & $P$ Value \\
\hline TPCS $(n=303)$ & -0.001 & 0.15 & 0.78 \\
IAR $(n=303)$ & -0.386 & 0.15 & 0.01 \\
Donor sex $(n=303)$ & 0.136 & 0.11 & 0.20 \\
Recipient sex $(n=303)$ & 0.026 & 0.12 & 0.85 \\
Recipient diagnosis $(n=303)$ & 0.006 & 0.03 & 0.45 \\
Donor liver type, DBD/DCD $(n=303)$ & 0.057 & 0.12 & 0.82 \\
Organ-perfusing support before & -0.487 & 0.24 & 0.10 \\
$\quad$ transplant $(n=303)$ & & & \\
Platelets before operation, 109/L & 0.0 & 0.001 & 0.83 \\
$\quad(n=301)$ & & & \\
Fibrinogen before operation, g/L & -0.038 & 0.02 & 0.13 \\
$\quad(n=299)$ & & & \\
Recipient age, years $(n=303)$ & 0.001 & 0.005 & 0.61 \\
Recipient BMI, kg/m $(n=303)$ & 0.0 & 0.012 & 0.92 \\
Donor age, years $(n=303)$ & -0.003 & 0.004 & 0.52 \\
Laboratory MELD $(n=303)$ & 0.034 & 0.007 & $<0.001$ \\
Donor last GGT, U/L $(n=300)$ & 0.001 & 0.001 & 0.33 \\
Hepatic vein pressure gradient, mm & 0.0 & 0.006 & 0.89 \\
$\quad$ Hg $(n=256)$ & & & \\
TPCS * IAR & & & 0.54
\end{tabular}

NOTE: Bolded values are significant. $R^{2}=0.224$ (adjusted $\left.R^{2}=0.127\right)$.

*Statistical interaction.

Multivariate analysis showed IAR to be an individual determinant on perioperative blood loss in LT. A possible explanation for this could be that the hepatic artery accounts for less total liver perfusion. Therefore, IAR may lead to a more controlled reperfusion and less blood loss.

This study also demonstrates that the use of a TPCS and the order of reperfusion used in LT do not have a significant influence on 1-year patient survival nor on 1-year graft survival. In general, DCD LT is associated with more complications and inferior outcomes compared with DBD LT. ${ }^{(23,30)}$ Even though the A+S+ group consisted of significantly more DCD LTs, we did not see a significant difference in patient and graft survival rates among the groups. Because the complication rate is higher with DCD LT compared with DBD LT, this may indicate a beneficial effect of both the use of IAR and TPCS. This is a very interesting finding that needs further research, especially because $>40 \%$ of LTs in the Netherlands are with a DCD liver.

The operative time between the patients with or without TPCS was almost identical. Creating a TPCS takes some extra time, but clearly these extra minutes are saved during the rest of the procedure. A possible explanation could be that the TPCS causes less venous congestion and resolves portal hypertension. This may make liver mobilization easier with less risk of blood loss, and because the liver hilum is fully transected, this may facilitate access to the dissection plane between the liver and the caval vein. Therefore, less time may be needed for hemostasis and explantation of the native liver. Furthermore, a better hemodynamic 


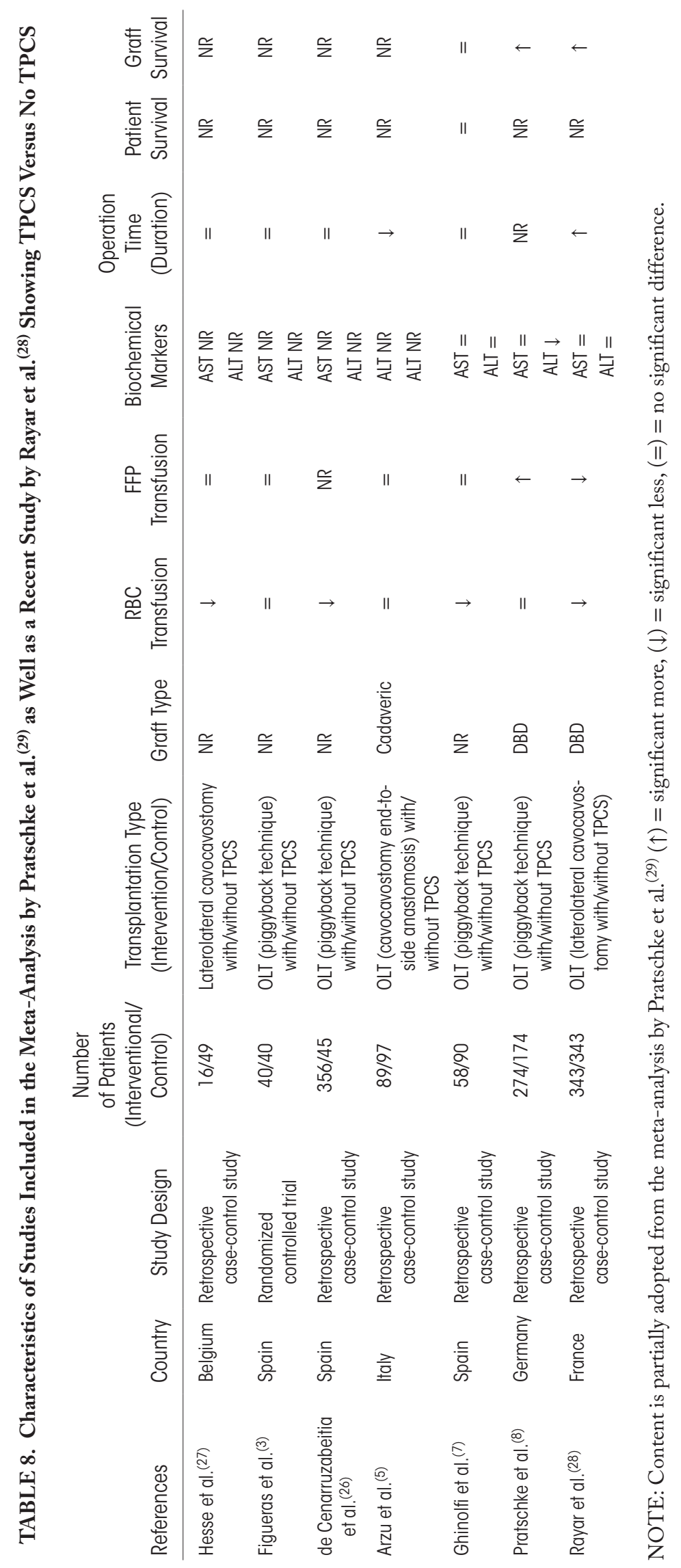


status during surgery due to less venous congestion may result in a more controlled situation. Since the introduction of the new protocol with IAR and TPCS, a significant increase in the number of recipients who did not receive any perioperative transfusion of $\mathrm{RBC} s$ was seen. This is in concordance with Figueras et al., ${ }^{(3)}$ who showed less decrease in cardiac output in the TPCS group $(P=0.05)$ as well as a greater diuretic output during the anhepatic phase $(P=0.005)$.

This study has some potential limitations. First, the nonrandomized character could bring a potential bias. Most controls were from the oldest cohort. However, some of the controls were from the most recent cohort, when the use of a TPCS and choice of reperfusion technique were according to the surgeon's preference. With the introduction of a new operative protocol, a selection bias is possible because the technique was new for some surgeons, and therefore, some preferred to use the previous surgical technique with initial portal reperfusion and no TPCS. Also, because of the retrospective character of the study, it is possible that other small changes in protocol have occurred during the study period, even though to our knowledge this is not the case. The major changes in protocol were the use of a TPCS and IAR. All relevant factors were included in the multivariate analysis to correct for these.

In conclusion, next to a lower laboratory MELD score, the use of IAR leads to significantly less perioperative blood transfusion. There was no significant interaction between IAR and TPCS. Furthermore, the use of a TPCS and/or IAR does not lead to increased operative time and is therefore a reasonable alternative surgical strategy.

\section{REFERENCES}

1) Filos KS, Kirkilesis I, Spiliopoulou I, Scopa CD, Nikolopoulou V, Kouraklis G, Vagianos CE. Bacterial translocation, endotoxaemia and apoptosis following Pringle manoeuvre in rats. Injury 2004;35:35-43.

2) Unno N, Uchiyama T, Yamamoto N, Inuzuka K, Sagara D, Konno H. Portal triad occlusion induces endotoxin tolerance: role of portal congestion. J Surg Res 2006;135:213-217.

3) Figueras J, Llado L, Ramos E, Jaurrieta E, Rafecas A, Fabregat J, et al. Temporary portocaval shunt during liver transplantation with vena cava preservation. results of a prospective randomized study. Liver Transpl 2001;7:904-911.

4) Tzakis AG, Reyes J, Nour B, Marino IR, Todo S, Starzl TE. Temporary end to side portacaval shunt in orthotopic hepatic transplantation in humans. Surg Gynecol Obstet 1993;176:180-182.

5) Arzu GD, De Ruvo N, Montalti R, Masetti M, Begliomini B, Di Benedetto F, et al. Temporary porto-caval shunt utility during orthotopic liver transplantation. Transplant Proc 2008;40:1937-1940.
6) Paugam-Burtz C, Kavafyan J, Merckx P, Dahmani S, Sommacale $\mathrm{D}$, Ramsay M, et al. Postreperfusion syndrome during liver transplantation for cirrhosis: outcome and predictors. Liver Transpl 2009;15:522-529.

7) Ghinolfi D, Martí J, Rodríguez-Laiz G, Sturdevant M, Iyer K, Bassi D, et al. The beneficial impact of temporary porto-caval shunt in orthotopic liver transplantation: a single center analysis. Transpl Int 2011;24:243-250.

8) Pratschke S, Meimarakis G, Bruns CJ, Kaspar M, Prix N, Zachoval R, et al. Temporary intraoperative porto-caval shunt: useless or beneficial in piggy back liver transplantation? Transpl Int 2013;26:90-98.

9) Audet M, Piardi T, Panaro F, Cag M, Habibeh H, Gheza F, et al. Four hundred and twenty-three consecutive adults piggy-back liver transplantations with the three suprahepatic veins: was the portal systemic shunt required? J Gastroenterol Hepatol 2010;25:591-596.

10) Kluger MD, Memeo R, Laurent A, Tayar C, Cherqui D. Survey of adult liver transplantation techniques (SALT): an international study of current practices in deceased donor liver transplantation. HPB (Oxford) 2011;13:692-698.

11) Muscari F, Suc B, Aguirre J, Di Mauro GL, Bloom E, Duffas JP, et al. Orthotopic liver transplantation with vena cava preservation in cirrhotic patients: is systematic temporary portacaval anastomosis a justified procedure? Transplant Proc 2005;37:2159-2162.

12) Ducerf C, Mechet I, Landry JL, DeLaRoche E, Berthoux N, Bourdeix $\mathrm{O}$, et al. Hemodynamic profiles during piggyback liver grafts using arterial or portal revascularization. J Am Coll Surg 2000;190:89-93.

13) Moreno C, Sabaté A, Figueras J, Camprubí I, Dalmau A, Fabregat J, et al. Hemodynamic profile and tissular oxygenation in orthotopic liver transplantation: influence of hepatic artery or portal vein revascularization of the graft. Liver Transpl 2006;12:1607-1614

14) Noun R, Sauvanet A, Belghiti J. Appraisal of the order of revascularization in human liver grafting: a controlled study. J Am Coll Surg 1997;185:70-73.

15) Sabaté A, Ferreres E, Valcárcel M, Dalmau A, Koo M, Fabregat J. Rocuronium profile during orthotopic liver transplantation: effect of changing the order of vascular clamp release at reperfusion of the hepatic graft. Transplant Proc 2010;42:1760-1762.

16) Sadler KM, Walsh TS, Garden OJ, Lee A. Comparison of hepatic artery and portal vein reperfusion during orthotopic liver transplantation. Transplantation 2001;72:1680-1684.

17) Walsh TS, Garden OJ, Lee A. Metabolic, cardiovascular, and acid-base status after hepatic artery or portal vein reperfusion during orthotopic liver transplantation. Liver Transpl 2002;8:537-544.

18) Broomhead RH, Patel S, Fernando B, O’Beirne J, Mallett S. Resource implications of expanding the use of donation after circulatory determination of death in liver transplantation. Liver Transpl 2012;18:771-778.

19) Durand F, Renz JF, Alkofer B, Burra P, Clavien PA, Porte RJ, et al. Report of the Paris consensus meeting on expanded criteria donors in liver transplantation. Liver Transpl 2008;14:1694-1707.

20) Meurisse N, Vanden Bussche S, Jochmans I, Francois J, Desschans B, Laleman W, et al. Outcomes of liver transplantations using donations after circulatory death: a single-center experience. Transplant Proc 2012;44:2868-2873.

21) Leithead JA, Tariciotti L, Gunson B, Holt A, Isaac J, Mirza DF, et al. Donation after cardiac death liver transplant recipients have an increased frequency of acute kidney injury. Am J Transplant 2012;12:965-975.

22) Blok JJ, Putter H, Rogiers $X$, van Hoek B, Samuel U, Ringers J, Braat AE; for Eurotransplant Liver Intestine Advisory Committee. 
Combined effect of donor and recipient risk on outcome after liver transplantation: research of the Eurotransplant database. Liver Transpl 2015;21:1486-1493.

23) Braat AE, Blok JJ, Putter H, Adam R, Burroughs AK, Rahmel $\mathrm{AO}$, et al.; for European Liver and Intestine Transplant Association (ELITA) and Eurotransplant Liver Intestine Advisory Committee (ELIAC). The Eurotransplant donor risk index in liver transplantation: ET-DRI. Am J Transplant 2012;12:2789-2796.

24) Dutkowski P, Oberkofler CE, Slankamenac K, Puhan MA, Schadde E, Müllhaupt B, et al. Are there better guidelines for allocation in liver transplantation? A novel score targeting justice and utility in the Model for End-Stage Liver Disease era. Ann Surg 2011;254:745-753.

25) Olthoff KM, Kulik L, Samstein B, Kaminski M, Abecassis M, Emond J, et al. Validation of a current definition of early allograft dysfunction in liver transplant recipients and analysis of risk factors. Liver Transpl 2010;16:943-949.

26) de Cenarruzabeitia IL, Lázaro JL, Bilbao I, Balsells J. Portocaval shunt throughout anhepatic phase in orthotopic liver transplantation for cirrhotic patients. Transplant Proc 2007;39: 2280-2284.

27) Hesse UJ, Berrevoet F, Troisi R, Mortier E, Decruyenaere J, Pattyn P, de Hemptinne B. Liver transplantation preserving the vena cava and a temporary portocaval shunt [in German]. Chirurg 1999;70:174-177.

28) Rayar M, Levi Sandri GB, Cusumano C, Locher C, HousselDebry P, Camus C, et al. Benefits of temporary portocaval shunt during orthotopic liver transplantation with vena cava preservation: a propensity score analysis. Liver Transpl 2017;23:174-183.

29) Pratschke $S$, Rauch $A$, Albertsmeier $M$, Rentsch M, Kirschneck $\mathrm{M}$, Andrassy J, et al. Temporary intraoperative porto-caval shunts in piggy-back liver transplantation reduce intraoperative blood loss and improve postoperative transaminases and renal function: a meta-analysis. World J Surg 2016;40:2988-2998.

30) Feng S, Goodrich NP, Bragg-Gresham JL, Dykstra DM, Punch JD, DebRoy MA, et al. Characteristics associated with liver graft failure: the concept of a donor risk index. Am J Transplant 2006;6:783-790. 